\title{
PINK PUFFER PROFILE in CEREBROVASCULAR DISEASES
}

\section{6-18 May 2018, Gothenburg}

Fiori Patrizia, Corbo Antonio, lorillo Luigi, Pelosi Chiara, Savino Patrizia, Bellizzi Gennaro, Dragonetti Carmela, Alberigo Marilisa, De Caro Monica, Guerriero Barbara, Pace Erminio, Raffa Mariangela, Giannetti Luigi Maria, Mazza Emerico, Tammaro Carmine, Monaco Antonio

S. Ottone Frangipane Hospital, ASL AV, Ariano irpino (AV) - University of Naples

\section{Introduction}

Cardiac biomarkers are increased in cerebrovascular conditions, especially in class III/IV, C/D New York Heart Association (NYHA) and American Cardiology Association (ACA) scales. Delta criterium allows differential diagnosis between acute and chronic conditions before the appearance of ischaemic signs at electrocardiogram (ECG) / echography (Fiori P. et al, 2017). The aim of our current study is to assess the impact of pulmonary emphysema.

\section{Materials and Methods}

So far, we recruited 63 acute strokes (AS, age 70,77 sd 14,36), 32 chronic cerebrovascular conditions (CCVD, age 73,62 sd 11,19), 25 other neuropsychiatric diseases (OND, age 50,88 sd 16,02). They underwent ECG and chest ray and/or CT at emergency department, blood withdrawal within 24 hours, echocardiography within one week.

\section{Results}

Preliminary results show significant higher levels of troponin ths (tro ths) and N-terminal-pro-Brain Natriuretic Peptide (NT-pro-BNP) levels in class III/IV, C/D NYHA and ACA AS and CCVD affected with emphysema (group A) compared to those without it (group B) (Fig. 1a and 1b). Glasgow Coma Scale (GCS) was lower and Modified Rankin Scale (MRS) was higher in the former compared to the latter (Fig. 2a, 2b). Central hyperventilation decreases $\mathrm{pCO}_{2}$ levels without compensating $\mathrm{pO}_{2}$ deficit (Fig. $3 a$ and $3 b$ ).

\section{Conclusions}

In "pink puffers" a mismatch between ventilation and perfusion is present. Low $\mathrm{pCO}_{2}$ may impair cerebral vasodilatation. Low $\mathrm{pO}_{2}$ reduces penumbra and enlarges necrotic ischaemic core. Structural pulmonary damage may account for concomitant chronic cerebrovascular sufferance, apoptotic neuronal death, lack of response to Non-Invasive Positive Pressure Ventilation.

\begin{tabular}{|c|c|c|c|c|}
\hline & CCVD & & AS & \\
\hline & No Emph & Emph & No Emph & Emph \\
\hline Tro ths pg/ml & 22,18 sd 20,31 & 50,85 sd 40,48 & 17,32 sd 15,59 & 32,8 sd 51,42 \\
\hline NT-pro-BNP pg/ml & $328,55 \mathrm{sd} 209,07$ & 2136,38 sd 2340,53 & 322,68 sd 349,81 & 5274,76 sd 8005,23 \\
\hline pO2 mmHg & 63,55 sd 19,75 & 62,85 sd 13,02 & 66,11 sd 14,18 & $68,29 \mathrm{sd} 11,7$ \\
\hline pCO2 mmHg & 44,36 sd 9,06 & 41,54 sd 11,07 & 41,43 sd 7,71 & $37,35 \mathrm{sd} 7,19$ \\
\hline day I GCS & 14 sd 1,9 & 13,46 sd 1,9 & 14,11 sd 1,69 & $11,73 \mathrm{sd} 3,02$ \\
\hline day VII GCS & $14 \mathrm{sd} 1,73$ & 13,46 sd 0,6 & 14,73 sd 0,02 & $12,68 \mathrm{sd} 2,51$ \\
\hline Delta GCS change & 0 sd 0,17 & $0 \mathrm{sd} 1,3$ & 0,62 sd 1,67 & $0,95 \mathrm{sd} 0,51$ \\
\hline pre-stroke MRS & 0,84 sd 1,38 & 1,89 sd 1,45 & 0,64 sd 1,23 & 1,36 sd 1,62 \\
\hline day VII MRS & $1,33 \mathrm{sd} 1,51$ & $2,28 \mathrm{sd} 1,56$ & 1,52 sd 1,54 & 2,89 sd 1,63 \\
\hline Delta MRS change & 0,49 sd 0,13 & 0,39 sd 0,11 & 0,88 sd 0,31 & $1,53 \mathrm{sd} 0,01$ \\
\hline
\end{tabular}
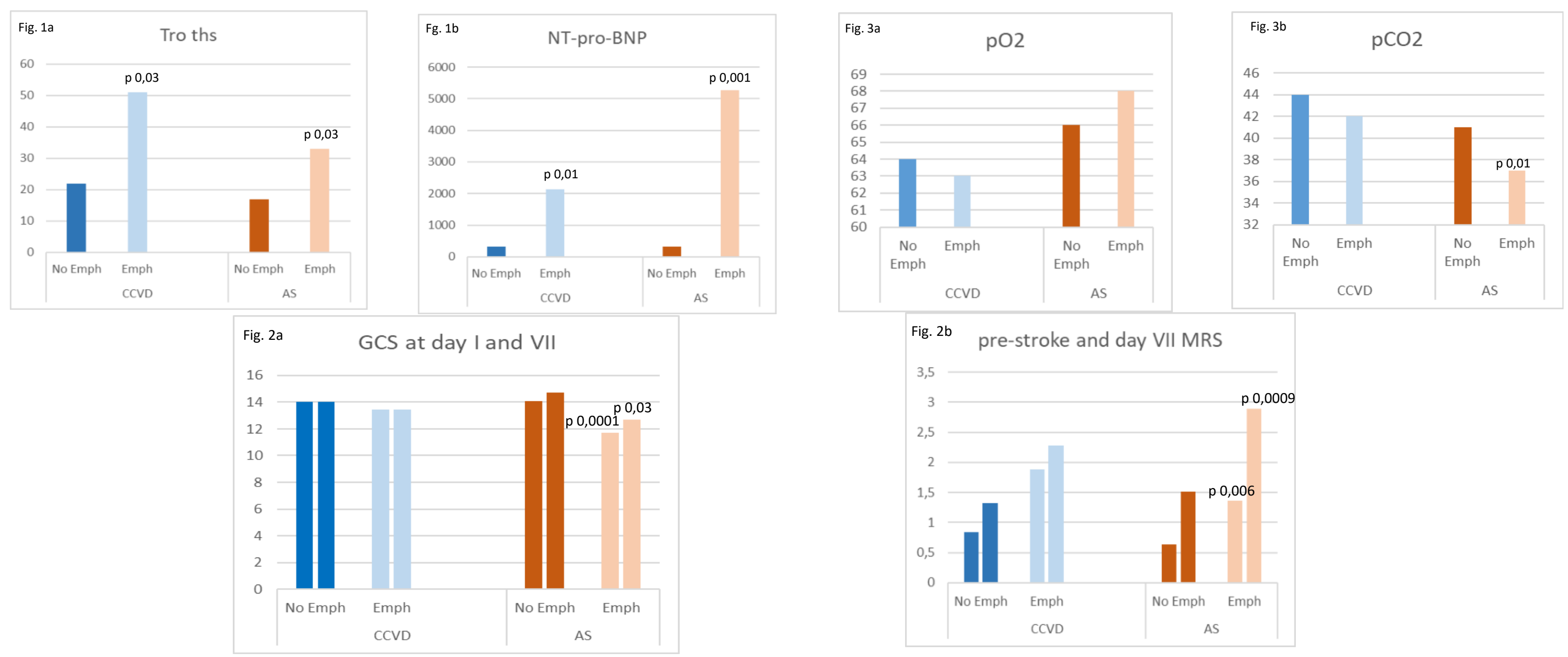